\title{
Our initial experience with the three layers with three-port approach for laparoscopic radical cystectomy
}

\author{
Xin Wang, Youlu Lu, Zhouting Tuo, Liangkuan Bi \\ Department of Urology, The Second Hospital of Anhui Medical University, Hefei, China
}

Videosurgery Miniinv 2022; 17 (1): 207-213

DOI: https://doi.org/10.5114/wiitm.2021.105572

\begin{abstract}
Introduction: Radical cystectomy $(R C)$ remains the gold standard for the treatment of recurrent high-risk non-muscle-infiltrating bladder cancer $(B C)$ and muscle-infiltrating $B C$. Currently, there is no uniform standardized procedure for laparoscopic radical cystectomy (LRC).

Aim: To share our initial experience with the three layers with three-port approach for laparoscopic radical cystectomy (TLTPA-LRC) and to investigate its safety and effectiveness.

Material and methods: Between April 2017 and March 2020, 32 patients with bladder tumors underwent TLTPA-LRC, pelvic lymph node dissection, and extracorporeal construction of the Studer neobladder. The basic characteristics of the patients, clinical pathology, and perioperative and follow-up data were analyzed. We also describe our step-by-step surgical technique for TLTPA-LRC.

Results: The median operation time was $278.5 \mathrm{~min}$ (range: 221-346 min), and the mean estimated blood loss was $233.4 \mathrm{ml}(102-445 \mathrm{ml})$. The rates of intraoperative blood transfusion and postoperative transportation to the intensive care unit after surgery were $12.5 \%$ and $100 \%$, respectively. Postoperative pathology showed 7 cases of T1, 20 cases of T2, and 5 cases of T3. Lymph node dissection and surgical margins were both negative. During a median follow-up of 13.5 months, 4 patients had early complications (< 30 days) and no patients had major complications (grade $\geq 3$ ). The patients are now alive without local metastasis and with satisfactory urinary control ability day and night.

Conclusions: Although the TLTPA-LRC approach requires a certain level of surgical proficiency, it is feasible and serves as a minimally invasive method for selected patients.
\end{abstract}

Key words: radical cystectomy, laparoscopy, bladder cancer, surgical treatment, urinary control ability.

\section{Introduction}

Radical cystectomy $(\mathrm{RC})$ remains the gold standard for recurrent high-risk non-muscle-infiltrating bladder cancer (BC) and muscle-infiltrating BC according to current guidelines [1]. In 1949, Whitmore and Marshall first reported the classical RC [2], and since then, this minimally invasive method has been the predominant surgical treatment for $\mathrm{BC}$. With the advent of laparoscopy, a new RC method has been developed. Parra et al. (1992) performed laparoscop- ic radical cystectomy (LRC) for the first time [3], and since then, LRC and urinary diversions have developed rapidly. Although some successful experiences in this area have been reported, there is no uniform standard for specific LRC procedures.

\section{Aim}

The aim of this paper is to describe our initial series of step-by-step LRC surgical techniques, the so-called three layers with three-port approach-LRC

\footnotetext{
Address for correspondence

Liangkuan Bi MD, Department of Urology, The Second Hospital of Anhui Medical University, Hefei, China,

e-mail: biliangkuan118@yeah.net
} 
(TLTPA-LRC). Based on the anatomical characteristics of the pelvic cavity and combined with previous clinical experience, the authors proposed a three-layer RC using a lateral approach. In other words, the lateral retroperitoneum is opened from the lateral side to expose the external iliac vessels (level 1), the anterior trunk of the internal iliac artery, the umbilical artery (level 2), and the ureter (level 3). Full exposure of important pelvic anatomical structures is achieved with the above design, and the surgeon can easily and programmatically cut off the blood supply to the bladder and the surrounding ligaments, and cut off the ureter. The vascular nerves and other tissues are fully "skeletonized", and five groups of important anatomical structures are exposed in turn from the outside to the inside, namely the genital femoral nerve, the external iliac artery and vein, the obturator nerve and blood vessels, the internal iliac blood vessels, and the ureter. The specific methods and preliminary results are reported below.

\section{Material and methods}

\section{Patients}

We reviewed and assessed the records of $32 \mathrm{pa}-$ tients with muscle-invasive or recurrent high-risk non-muscle-invasive $B C$ with negative staging assessment who underwent TLTPA-LRC at our hospital from April 2017 to March 2020. Patient characteristics (age, sex, body mass index, clinical stage, and size of bladder tumor) were evaluated. All patients underwent B-mode ultrasound, computed tomography (CT), and/ or magnetic resonance imaging (MRI) preoperatively to determine tumor locations and pelvic lymph node metastasis. All patients underwent cystoscopy or diagnostic electrosurgical resection of tumor tissue for examination. The pathological diagnosis was invasive bladder tumor or advanced noninvasive bladder tumor. CT, MRI, and other imaging examinations showed no distant metastasis, and significant pelvic lymph node involvement was suspected in 1 case. BC was classified according to the 2017 American Joint Committee on Cancer staging manual [4]. Preoperatively, it was confirmed that patients had good urinary control ability and no intestinal lesions or history of intestinal resection. All patients underwent LRC, pelvic lymph node dissection, and extracorporeal construction of the Studer orthotopic neobladder in accordance with the step-by-step procedure. All procedures were performed by a single surgeon (LK Bi) with advanced lap- aroscopic skills. The study was approved by the ethics committee of the Second Hospital of Anhui Medical University. All patients signed written consent to allow the use of their data.

\section{Preoperative preparation}

General preoperative preparation included preoperative skin preparation, fasting, and water enema. The patients were given a fluid diet on the day before surgery, and an enema and water fasting the night before the operation. Oral antibiotics were taken for 1 day before surgery to avoid the presence of bowel anaerobic bacteria and Gram-negative bacilli. These antibiotics, such as metronidazole and levofloxacin, were used for bowel preparation and intraoperative intestinal tract rupture during the enteric suture stage.

\section{Surgical technique}

\section{Anesthesia and position}

The patient was placed in the supine position with a $30^{\circ}$ tilt to provide a lower head setting, and with buttocks raised and shoulders fixed. Patients underwent endotracheal intubation, general anesthesia, and intoxication. The operator was positioned on the left side of the patient. The assistant was positioned on the right side to deliver the laparoscopic instrument and assist in the extracorporeal construction of the neobladder, and person who held the scope was positioned on the side of the patient's head.

\section{Trocar configuration}

The first puncture point (point A) was the observation hole (Figure 1), which was $2 \mathrm{~cm}$ above the umbilicus. The incision made was about $1 \mathrm{~cm}$, the pneumoperitoneum needle was inserted, abdominal pressure was adjusted to about $15 \mathrm{~mm} \mathrm{Hg}(1 \mathrm{~mm} \mathrm{Hg}$ $=0.133 \mathrm{kPa}$ ), and then a $10-\mathrm{mm}$ observation trocar was inserted at the first puncture point to observe the abdominal pelvic cavity by laparoscope, and eventually the remaining two trocars were placed under laparoscopic observation (points B and C). A $1-\mathrm{cm}$ incision was made next to the bilateral rectus abdominis muscles, and $12-\mathrm{mm}$ and $5-\mathrm{mm}$ trocars were placed near the umbilicus.

\section{Dissociated bilateral ureter}

Open lateral peritoneum was performed using our specific lateral three-layer approach (Figure 2). 


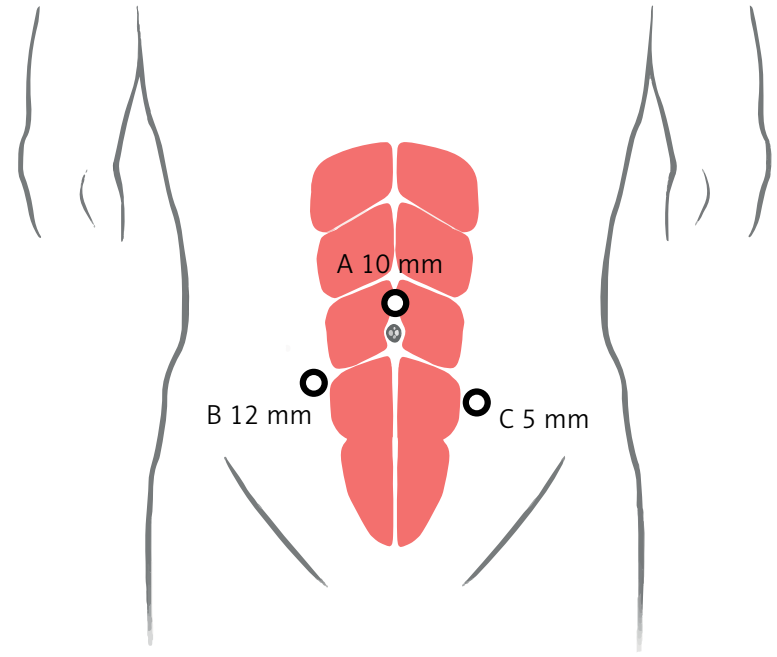

Figure 1. Port placement and docking for the three layers with the three-port approach for laparoscopic radical cystectomy. A - The $10-\mathrm{mm}$ port for the observation hole, at about $2 \mathrm{~cm}$ above the umbilicus. B - The $12-\mathrm{mm}$ port for the main operation hole, at about 3-4 cm below the umbilicus of the right rectus muscle. $\mathrm{C}-$ The 5-mm port for the assisted operation hole, at about $5-7 \mathrm{~cm}$ below the umbilicus of the left rectus muscle

The lateral peritoneum was opened along the lateral side of the external iliac vessels and the medial side of the spermatic vein. The upper part started from the position where the ureter crossed the iliac blood vessel, and the lower part reached the position of the vas deferens near the inner ring mouth. For enlarged lymphadenectomy, the upper aortic bifurcation position was reached. The first layer, the external iliac vessel layer, was exposed, and the peritoneum was lifted using separation forceps. The ultrasonic knife was used to push the peritoneum along the inside of the external iliac arteries and veins, and the vas deferens was broken downward to reveal the bladder side space and expand upward. This first stage of surgery ended with exposure of the external iliac vessel. The second layer, the internal iliac vessel layer, was revealed and the peritoneum was opened continuously, while the internal iliac artery was found. Then, the internal iliac artery was raised, and the anterior trunk of the internal iliac artery was exposed and then clamped. This second stage of surgery ended with exposure of the internal iliac vessels. The third layer, the ureter layer, was exposed, and the internal iliac artery was lifted,

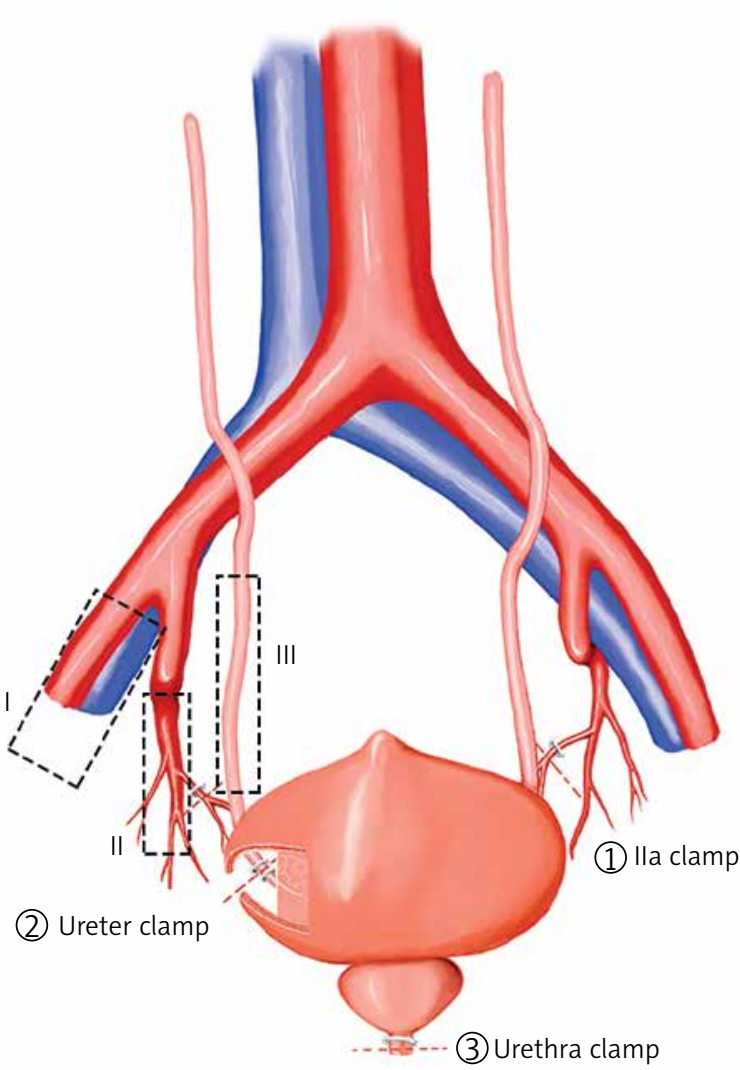

Figure 2. Sketch map for the three layers technique. I - the external iliac vessel layer, II - the internal iliac vessel layer, III - the ureter layer. The main procedure includes the following steps. (1) The lateral peritoneum is opened along the lateral side of the external iliac vessels and the internal iliac artery (IIA) is clamped with a medium-sized Hem-o-lock. (2) The ureter is found below the root of the umbilical artery, and then the peritoneum is pushed inward to expose the ureter. The ureter is clamped with a medium-sized Hem-o-lock. (3) The urethra is clamped with a large Hem-o-lock

and the peritoneum pushed inwards with the umbilical artery as the marker. Meanwhile, the ureter was found below the root of the umbilical artery, and then the peritoneum was pushed inward to expose the ureteral layer. The same method could be adopted to expose the left side. In order to better expose the common iliac blood vessels, the ureter should be pushed inward and upward as far as possible to expose the pelvic lymph nodes and protect the ureteral blood supply to the greatest extent and reduce the incidence of postoperative ureteral stricture. 


\section{Dissociated bilateral ligaments of the bladder and prostate}

The bladder was lifted up, and the lateral bladder space (between the bladder and pelvic side wall) was fully dissociated. The lateral bladder ligament was exposed and clamped with Hem-o-lock clamps. After that, it was cut off with an ultrasonic knife. The bladder was lifted using an elastic separating plier, the Douglas pouch was revealed, and then the peritoneum was cut apart. The seminal vesicles were held up, and the Denonvilliers' fascia was cut through with an ultrasonic knife until the apex part of the prostate was separated. Then, the ligament of the prostate was clamped with Hem-o-lock clamps. The umbilical median ligament, paramedian ligament, and peritoneal reflex were cut to expose the pelvic cavity fully. Then, we continued to dissect the apex of the prostate and exposed the pubic prostate ligament and pelvic fascia reflex. The pelvic fascia reflex and pubic prostate ligament were dissected by means of close attachment to the levator anal muscle and pubis.

\section{Removal of the bladder and prostate from the urethra}

The dorsal venous complex was dissected with scissors near the suture line, close to the prostatic apex, until the urethra was separated, and the urethral length was retained long enough to maximize postoperative urinary control ability. The urethra was clipped with a large Hem-o-lock and then cut. The bladder and prostate were removed, and specimens were collected in specimen bags.

\section{Dissected pelvic lymph node and extracorporeal} construction of the Studer orthotopic neobladder

Pelvic lymph node dissection and extracorporeal construction of the Studer orthotopic neobladder were performed using the standard procedure $[5,6]$ for each patient.

\section{Postoperative treatment and follow-up}

Bladder irrigation was started on the first day after surgery (200-250 $\mathrm{ml}$ of normal saline every 6$8 \mathrm{~h}$ ). The patient was instructed to get out of bed as soon as possible, and was given a liquid diet after the gastrointestinal tract resumed ventilation, which was gradually changed to a normal diet. After ob- serving a pelvic drainage volume $<100 \mathrm{ml} /$ day that was gradually decreasing, the drainage tube was pulled out. Neobladder cystography was performed 3-4 weeks after surgery. If no leakage was found, the catheter was removed. After a week of urination training, patients were instructed to urinate once every $2-3 \mathrm{~h}$ during the day (every $4 \mathrm{~h}$ at night) and gradually extend the interval between urination to increase the capacity of the new bladder. During this time, patients were advised not to urinate immediately when they felt incontinent.

Patients were followed up via telephone and outpatient clinic appointments. Routine blood, urine, blood gas biochemistry, and urinary system B-mode ultrasound examinations were performed monthly for 3 months after the operation. Follow-up focused on urinary incontinence and quality of life. The National Cancer Network Comprehensive Functional Assessment of Cancer Treatment Functional Bladder Symptom Index 18 (NCCN-FACT-FBISI18) questionnaire was used to evaluate the quality of life of patients.

\section{Results}

All patients underwent LRC, pelvic lymph node dissection, and extracorporeal construction of the Studer orthotopic neobladder with the same programmed procedure without additional trocars. The median operation time was $278.5 \mathrm{~min}$ (221-346 min), and mean estimated blood loss was $233.4 \mathrm{ml}$ (102$445 \mathrm{ml})$. The rates of intraoperative blood transfusion and postoperative transformation to intensive care units after surgery were $12.5 \%$ and $100 \%$, respectively. Postoperative pathology showed 7 cases of T1, 20 cases of T2, and 5 cases of T3. Lymph node dissection and surgical margins were both negative. During the short-term follow-up, 1 patient was admitted to the hospital for urethral stricture and underwent urethral dilatation regularly, while 2 patients underwent bilateral renal puncture and fistula plus anterograde catheterization due to bilateral ureteral stricture, and another underwent hemodialysis because of renal failure. The patients are all alive without local metastasis, and with satisfactory urinary control ability day and night (Tables I and II).

\section{Discussion}

In the 1940s, the survival rates and oncological outcomes of RC were poor [7]. In 1948, Jewett and 
Lewis reported their experience of RC for the first time [8]. With the development of surgical endoscopy technology, the concepts of minimally invasive $R C$ (MIRC), such as LRC and robot-assisted laparoscopic radical cystectomy (RA-LRC), have been increasingly accepted for treatment of $\mathrm{BC}[9,10]$.

Based on the anatomical features of the pelvic cavity and our previous clinical experience, a lateral three-layered approach was proposed by our institution (Photo 1). Briefly, the lateral posterior perito- neum was opened to expose the external iliac vessels (level 1), the anterior trunk of the internal iliac artery, the umbilical artery (level 2), and the ureter layer (level 3) in sequence. In this way, the vital pelvic anatomy was fully exposed, and five groups of important anatomic structures were dissected successively from the outside and inside, namely the reproductive femoral nerve, external iliac blood vessel, obturator nerve and blood vessels, internal iliac blood vessels, and the ureter.
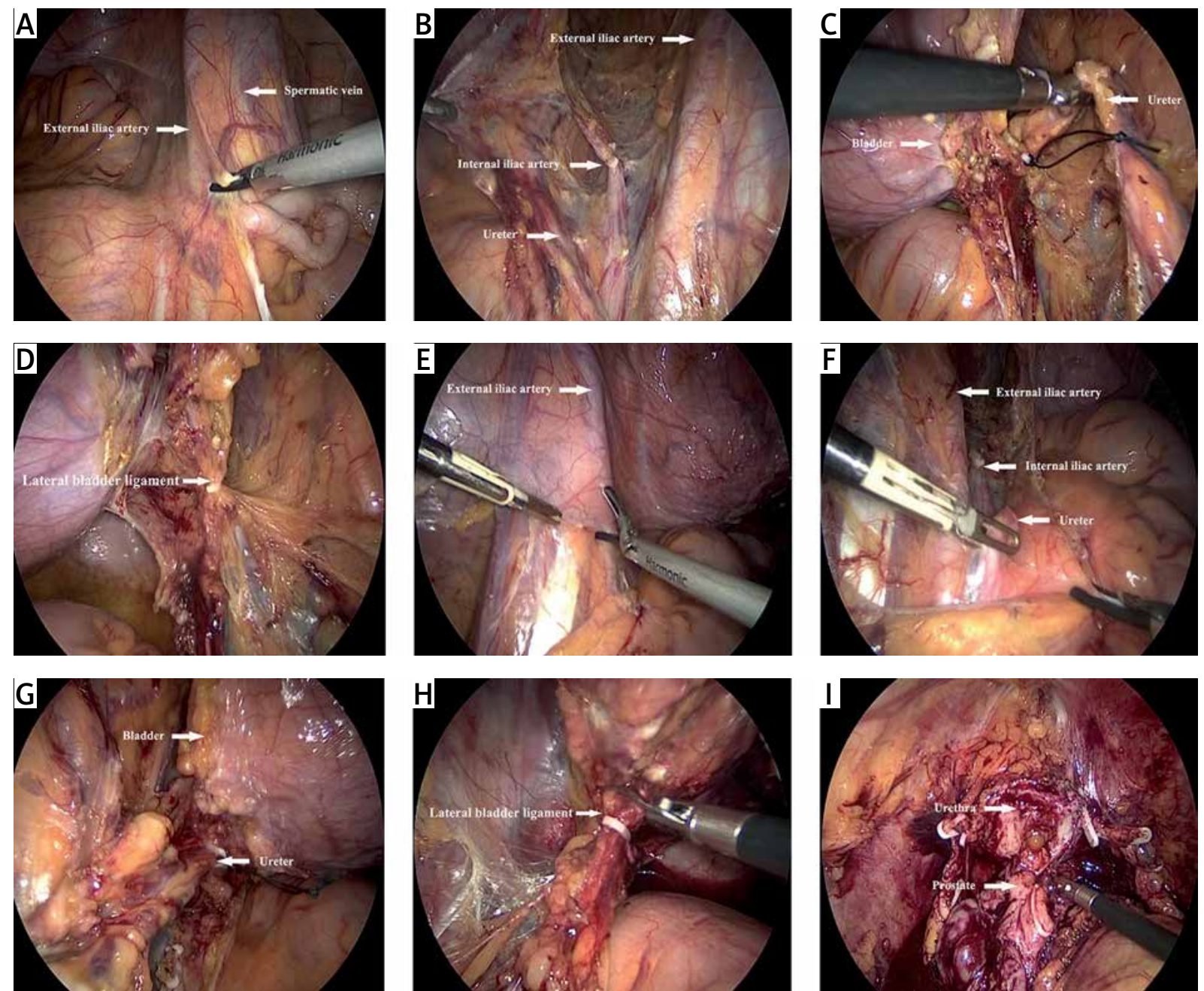

Photo 1 . The critical procedure of the lateral three layers laparoscopic radical cystectomy technique. The right ureter is dissociated. A - The right lateral peritoneum is opened along the lateral side of the external iliac vessels and the medial side of the spermatic vein. $\mathbf{B}$ - The right three layers are exposed, and the right internal iliac artery (IIA) is clamped. C - The right ureter is clipped with a corded Hem-o-lock and then cut off. D - The right lateral bladder ligament is clamped and cut off. The left ureter is dissociated. E - The left lateral peritoneum is opened along the lateral side of the external iliac vessels and the medial side of the spermatic vein. $\mathbf{F}$ - The left three layers are exposed, and the left IIA is clamped. G - The left ureter is clipped with a corded Hem-o-lock and then cut off. $\mathbf{H}$ - The left lateral bladder ligament is clamped and cut off. The urethra is cut off completely. I - The urethra is cut off completely, and the bladder is cut off 
Classical LRC usually involves pelvic lymphadenectomy before removal of the bladder. Although this method is convenient in terms of surgical tech-

Table I. Clinical characteristics of all 32 patients

\begin{tabular}{|lc|}
\hline Characteristic & Result \\
\hline Age [years] median (range) & $60.5(41-73)$ \\
\hline Sex (male/female) & $20 / 12$ \\
\hline BMI $\left[\mathrm{kg} / \mathrm{m}^{2}\right]$ mean (range) & $22.7(18.9-27.3)$ \\
\hline Tumor size [cm] mean (range) & $3.7(1.2-6.3)$ \\
\hline pT stage, $n(\%)$ & $7(21.9)$ \\
\hline T1 & $20(62.5)$ \\
\hline T2 & $5(15.6)$ \\
\hline T3-T4 & $25(78.1)$ \\
\hline pN stage, $n$ (\%): & $4(12.5)$ \\
\hline N0 & $3(9.4)$ \\
\hline N1 & $30(93.8)$ \\
\hline N2
\end{tabular}

$B M I-$ body mass index.

Table II. Perioperative data

\begin{tabular}{|lc|}
\hline Characteristic & Result \\
\hline Operative time [min] median (range) & $278.5(221-346)$ \\
\hline Estimated blood loss [ml] mean (range) & $233.4(112-315)$ \\
\hline Intraoperative blood transfusion, $n$ (\%) & $4(12.5)$ \\
\hline Postoperative transformation to ICU, $n$ (\%) & $32(100)$ \\
\hline $\begin{array}{l}\text { Time to full ambulation [days] median } \\
\text { (range) }\end{array}$ & $3.0(2.5-4)$ \\
\hline Time to oral feeding [days] median (IQR) & $1.5(1.5-3)$ \\
\hline $\begin{array}{l}\text { Postoperative hospital stay [days] mean } \\
\text { (range) }\end{array}$ & $11.9(7-16)$ \\
\hline \begin{tabular}{l} 
Clavien-Dindo grade, $n$ (\%): \\
\hline 1
\end{tabular} & 23 \\
\hline 2 & 0 \\
\hline 3 & 0 \\
\hline 4 & $9(28.1)$ \\
\hline Adjuvant chemotherapy received, $n(\%)$ & 9 \\
\hline
\end{tabular}

ICU - intensive care unit, IQR - interquartile range. ${ }^{a}$ According to the Clavien-Dindo classification of surgical complications [16]. nique, postoperative pelvic reconstruction is often unsatisfactory due to the destruction and lack of normal anatomical structure levels after lymphatic dissection. Our initial series focuses on opening the outer peritoneum, gradually freeing the iliac vessels along the normal anatomical level, finding and ligating the ureter, removing the bladder, and finally performing lymph node dissection. Through the accumulation of experience, we believe that this is helpful for protecting and rebuilding the integrity of the peritoneum and preventing postoperative intestinal obstruction.

Although the pelvic cavity is a symmetrical structure, there are slight differences between the right and left sides [11]. Our initial experience was that the key point of the right three-layer method was to expose and ligate the umbilical artery first, which is more conducive to dissociating the ureter and protecting its blood supply. The left side should be given priority to expose and clip the ureter because the umbilical artery is easy to locate when the ureter is dissociated.

Relatively popular trocar placement methods include the four-port method and the five-port method. The four-port method features fewer surgical incisions; however, it lengthens the operation time significantly and is not conducive to postoperative bowel recovery. The moderate exposure of the fiveport method is sufficient to some extent, and the operation time is relatively short $[12,13]$. However, we used the three-port approach, and our initial intention was to make the incision more conducive to improve the aesthetic appearance of patients and more consistent with the minimally invasive concept. However, without a doubt, this method increases surgical difficulty and requires a high level of laparoscopic surgical ability. Therefore, it is proposed that this novel method may be suitable only for surgeons with competency in laparoscopy.

The three-layered approach was initiated by Dr. $\mathrm{Bi}$ in 2018 [14]. At first, this method was proposed for simplifying the pelvic lymph node dissection in several pelvic procedures involving malignant tumors, such as RC, radical prostatectomy for high-risk prostate cancer, and suspected pelvic lymphatic metastasis for penile cancer [15]. With the continuous accumulation of surgical experience using this approach, it was found that it is feasible to apply it to RC. Our study has indicated that RC is feasible and repeatable by following a standardized surgical procedure. 
Although the detailed technique of TLTPA-LRC has been discussed comprehensively, several limitations should be noted. First, our experience has mainly focused on RC. Whether or not there are differences in efficiency in different methods of urinary diversion remains uncertain. Second, only a few patients were included in our study. Finally, this type of laparoscopic technique needs relevant proficient skills, and reports of the experience of other surgeons are necessary to confirm its repeatability before it can be widely accepted.

\section{Conclusions}

TLTPA-LRC is feasible and safe for patients. Further studies with larger sample sizes and longer follow-up times are required to confirm the findings of our investigation.

\section{Acknowledgments}

This work was supported by the National Natural Science Foundation of China (grant number 81572507).

Xin Wang and Youlu Lu contributes equally to this work.

\section{Conflict of interest}

The authors declare no conflict of interest.

\section{References}

1. Alfred WJ, Lebret T, Compérat EM, et al. Updated 2016 EAU guidelines on muscle-invasive and metastatic bladder cancer. Eur Urol 2017; 71: 462-75.

2. Marshall VJ, Whitemore WJ. A technique for the extension of radical surgery in the treatment of vesical cancer. Cancer 1949; 2: 424-8.

3. Parra RO, Andrus CH, Jones JP, Boullier JA: Laparoscopic cystectomy: initial report on a new treatment for the retained bladder. J Urol 1992; 148: 1140-4.

4. Amin MB, Edge S, Greene F, et al. AJCC cancer staging manual. Springer International Publishing 2017.

5. Desai MM, Berger AK, Brandina RR, et al. Robotic and laparoscopic high extended pelvic lymph node dissection during radical cystectomy: technique and outcomes. Eur Urol 2012; 61: 350-5.

6. Hautmann RE, Botto H, Studer UE. How to obtain good results with orthotopic bladder substitution: The 10 commandments. European Urology Supplements. The 2nd World Congress on Controversies in Urology 2009; 8: 712-7.

7. Whitmore WF. Bladder cancer. CA Cancer I Clin 1978; 28: 170-7.

8. Jewett HJ, Lewis EL. Infiltrating carcinoma of the bladder: curability by total cystectomy. J Urol 1948; 60: 107-18.
9. Bochner BH, Dalbagni G, Marzouk KH, et al. Randomized trial comparing open radical cystectomy and robot-assisted laparoscopic radical cystectomy: oncologic outcomes. Eur Urol 2018; 74: 465-71.

10. Aboumarzouk OM, Hughes O, Narahari K, et al. Safety and feasibility of laparoscopic radical cystectomy for the treatment of bladder cancer. J Endourol 2013; 27: 1083-95.

11. Williams SB, Kamat AM, Lamm DL. Pelvic recurrence after radical cystectomy: a call to arms. BJU Int 2015; 116: 172-3.

12. Huang J, Liu H, Lin T, et al. Status and prospect of laparoscopic radical cystectomy: ten years experience summary and systematic literature analysis. Chinese J Urol 2012; 805-9.

13. Gill IS, Fergany A, Klein EA, et al. Laparoscopic radical cystoprostatectomy with ileal conduit performed completely intracorporeally: the initial 2 cases. Urology 2000; 56: 26-29, 29-30.

14. Bi L, Ding D, Wang J, Yu D. Application of three-layer lateral approach in laparoscopic pelvic lymphadenectomy. I Sun Yat-Sen Univ 2018; 3: 463-6.

15. Protzel C, Alcaraz A, Horenblas S, et al. Lymphadenectomy in the surgical management of penile cancer. Eur Urol 2009; 55: 1075-88.

16. Dindo D, Demartines N, Clavien PA. Classification of surgical complications: a new proposal with evaluation in a cohort of 6336 patients and results of a survey. Ann Surg 2004; 240: 205-13.

Received: 30.01.2021, accepted: 7.03.2021. 